\title{
SOCIAL SENSOR: AN ANALYSIS TOOL FOR SOCIAL MEDIA
}

\author{
Chun-Hsiao Wu \\ National Chengchi University \\ 64, Sec. 2, Zhi-Nan Rd., Taipei, Taiwan \\ shaldrem@gmail.com \\ Tsai-Yen Li \\ National Chengchi University \\ 64, Sec. 2, Zhi-Nan Rd., Taipei, Taiwan \\ li@nccu.edu.tw
}

\begin{abstract}
In this research, we propose a new concept for social media analysis called Social Sensor, which is an innovative design attempting to transform the concept of a physical sensor in the real world into the world of social media with three design features: manageability, modularity, and reusability. The system is a case-centered design that allows analysts to select the type of social media (such as Twitter), the target data sets, and appropriate social sensors for analysis. By adopting parameter templates, one can quickly apply the experience of other experts at the beginning of a new case or even create one's own templates. We have also modularized the analysis tools into two social sensors: Language Sensor and Text Sensor.

A user evaluation was conducted and the results showed that usefulness, modularity, reusability, and manageability of the system were all very positive. The results also show that this tool can greatly reduce the time needed to perform data analysis, solve the problems encountered in traditional analysis process, and obtained useful results. The experimental results reveal that the concept of social sensor and the proposed system design are useful for big data analysis of social media.
\end{abstract}

Keywords: Social Sensor, Social Media, Data Science, Big Data Analysis, Automated Analysis 


\section{INTRODUCTION}

\subsection{Research Motivation and Purpose}

The rise of social media networks established a new style of network structure, and the policy of open data has led to data analysis of the global social media frenzy. The current rapid growth of a huge amount of social media data has led many people and resources invested in the task to collect, filter, store, process, and manage a huge amount of complex raw data quickly. In addition, the growth rate has been much greater than the speed that a human expert can analyze.

Ben Lorica ${ }^{1}$ pointed out that data scientists tend to use a variety of tools, often across different programming languages, to process big data. Workflows that involve many different tools require a considerable amount of context-switching, which affects productivity and impedes reproducibility. Similarly, the analysis workflow in a data science team usually adopts a sub-role, sub-field approach to the entire analytical work. In the team, some engineers are responsible for crawl data, and then transfer the data to processing data operations performed by other engineers. Output data is then explored and established by data analysts, which is then followed by data visualization staff to present analysis results to the domain expert for interpretation.

In this research, we studied the data analysis process of a research team at National Chengchi University who are analyzing social media. We used the case study "2012 Taiwan presidential election" "to illustrate the problems in a typical analysis process. These challenges include the unmatched speed of data analysis with the speed of data collection, independent and fragmented analysis steps, labor intensive manual analysis, manual file exchanges, lack of data and case management tools, difficulty in maintaining domain expertise, high restart costs, long waiting times, etc.

As such, in this research we propose a new concept for social media analysis called "Social Sensor," which is an innovative design attempting to transform the concept of a physical sensor in the real world into the world of social media with three design features: manageability, modularity, and reusability. This tool is used to help users collect a variety of social media data for rapid analysis and visual presentation. By developing the concept of social sensors for a massive amount of social media analyses, we aim to provide an analytical tool for application in social science studies addressing policy making, marketing, and the design of an intelligence interface. 


\subsection{Concept and Design Goals}

This research attempts to bring the concept of physical sensors to the virtual world of social media, shown in Figure 1. Physical sensors in a real-world environment are like social sensors in the social media environment, and they all produce a tremendous amount of environmental data. Physical sensors measure and produce physical or chemical data, such as light, heat, temperature, and humidity. The equivalent collected from social media by social sensors may include tweets, users, locations, etc. More specifically, examples of physical sensors include a luminosity sensor, temperature sensor, etc., whereas social sensors may include language sensors, text sensors and the like. Social sensors also function similarly to physical sensors because different sensors are designed to have their own sensing tasks and applications. A social sensor is implemented with various analytical methods and generates derived data for a specific analytical task.

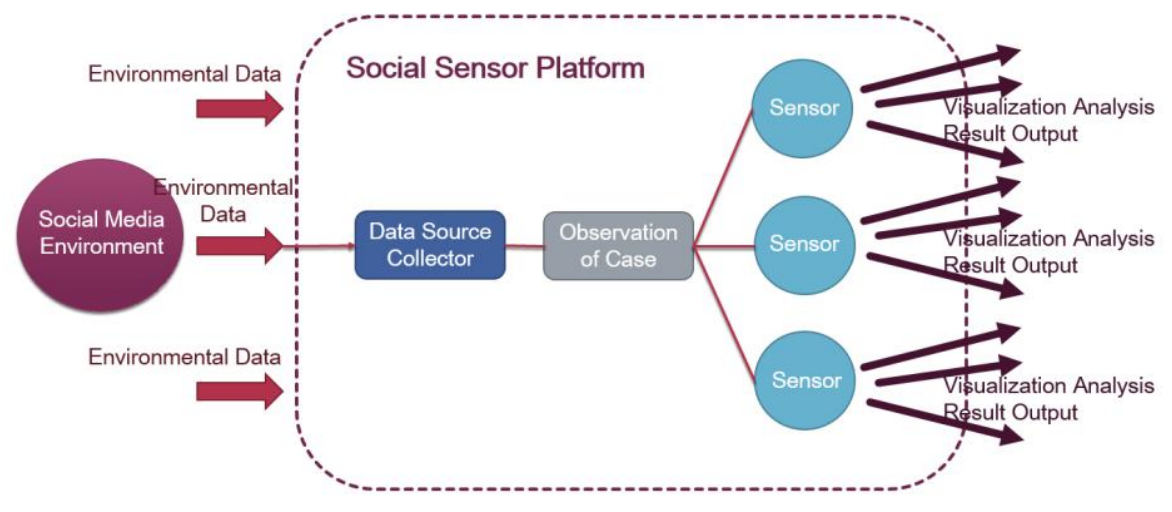

Figure 1. Social sensor conceptual model

In addition, some physical sensors may contain parameter settings that can be used to adapt to different applications, and the settings of these parameters usually come from prior knowledge and experience. Similarly, in the social sensor design, through parametric design, the same sensor can be customized to meet the needs of a specific application. Experience can then be passed on from one case to another in a different setting. This can be done by copying the set parameters as initial setting, thus making it unnecessary to start from scratch with trial and error.

\section{RELATED RESEARCH}

\subsection{Social Media and Social Network Analysis}

Social Media on the Internet is defined in Kaplan and Haenlein's research $^{3}$ as a group of applications featuring User Generated Content (UGC) and media richness. Examples of social media includes Twitter, Facebook, 
YouTube, Blog, Plurk, Flickr, etc. It can be viewed broadly as a service platform for people to share with each other ideas, insights, experiences and perspectives through various forms of media. These applications are built on the concepts and techniques of Web 2.0 and allows User Generated Content (UGC) to be established and exchanged. UGC is usually used to describe various forms of media content that are publicly available and created by end-users ${ }^{4}$.

Social Network Analysis is a set of norms and methods to study the relationship between structure and properties. Cohen ${ }^{5}$ put forth that social media can be called an outlet for strategy and broadcasting while social networking is a tool and a utility for connecting with others. Yuan et al. ${ }^{6}$ use semantic network analysis to calculate the frequency of words and phrases, the co-occurrence of conditions, and the distance between words. The algorithm divides the data into different network clusters and uses a semantic network diagram to do the study. Kwak et al. ${ }^{7}$ use statistics, interactive link analysis, and a separation index to investigate the Tweeter environment and its characteristics in terms of information propagation.

In addition, many tools exist for social media analysis. For example, yourTwapperkeeper is a tool can be used to track more than one set of keywords or hashtags. Others include the statistical package for Microsoft Office Excel, text processing software packages such as Leximancer and Wordstat, and network analysis and visualization tools such as Gephi. However, no single system or platform can serve as the best tool for all users since different application domains have different needs.

\subsection{Application of Social Network Analysis}

The techniques of Social Network Analysis (SNA) are quite extensive and its applications are also quite diverse. For example, some common techniques include data fusion and data mining, network communication modeling, user attributes and user behavior analysis, interaction analysis based on location, social sharing and filtering, recommendation systems, etc. These techniques can be applied to business practices, such as customer interaction and analysis, marketing, and business intelligence. It can also be applied to information collection and consolidation, counter-intelligence, and law enforcement activities. There are also studies on food safety that make use of SNA techniques to build a query platform for plasticizer tests. Politics have also utilized these tools, for example, Burgess and Bruns ${ }^{8}$ used Tweeter data to study the 2010 Australian Prime Minister Election. Others have used SNA techniques to study catastrophic events. For example, Vieweg et $\mathrm{al}^{9}$. investigated how to capture relevant important messages by information extraction technologies. Finally, Shih ${ }^{10}$ used machine learning 
techniques to process real-time new media content for automatic classification.

\subsection{Social Sensors}

"Social Sensor" is a new concept derived from physical sensors. However, the concept has been defined by various researchers looking at social media and there are different definitions. In this research, the innovative concepts and design of our social sensor are quite different from those previously proposed.

Vasudevan ${ }^{11}$ et al. applied the concept of a social sensor to television programs. They utilized a social sensor design through the discussion and dissemination of real-time social media (such as Tweeter) to determine the hot peak point of social media in order to recommend a given TV program. Although this mechanism can potentially lead a large amount of social network users to participate in a real-time TV show, it is only suitable for interstitial viewing.

Sakaki $^{12}$ et al. used the field characteristic of Twitter to study real-time interactions during an earthquake event. They used a keyword classifier for tweets, the number of words, and their contexts to build a model for computing the event location center and trace probability. They considered each Twitter user as a sensor and applied Kalman filtering and particle filtering for location estimation. Following this work, in 2013, Sakaki ${ }^{13}$ et al. proposed a method to extract real-time traffic information from social sensors in social media. Social sensors extracted information from social media through text-based classification, geographical coordinates, and important traffic events to provide real-time driving advice.

The aforementioned social sensors attempted to use the real-time characteristics of social media and to transform the given data into useful information in specific applications. The main body of a social sensor can be referred to as a data analysis method or to the user of social media. In contrast, the concept of a social sensor in this research does not only refer to the design of a specific function of a sensor, but also includes features of pluggability and manageability. This allows one to construct a platform for a particular observation of social media from a wide range of sensors. More specifically, in this research, our social sensors have three features: manageability, modularity, and reusability. These features satisfy the needs of multidisciplinary analysis, plug-and-play, experience transfer through reuse, and case management. 


\section{SYSTEM CONCEPTS AND DESIGN}

\subsection{System Concept}

In this research, we used the research team looking at social media analysis at National Chengchi University as an example to understand the typical analysis process and to identify current problems in the process. The process typically consists of three phases: data preparation, data analysis, and data presentation. The data preparation phase typically includes the following steps: event occurrence, keyword collection, data storage and management, waiting for harvest. The data analysis phase includes data preprocessing and data analysis while the data presentation phase includes data visualization and interpretation. We have used the concept of social sensor to streamline the steps in these three phases. Each sensor adopts a combination of the analytical methods in these steps and works independently. In addition, we try to separate data sets from observation cases for better manageability and reusability. In other words, the system uses a case-centered design, shown in Figure 2, which allows analysts to select the type of social media (such as Twitter), the target data sets, and appropriate social sensors for analysis. By adopting parameter templates, one can quickly apply the experience of other experts at the beginning of a new case or even create one's own templates.

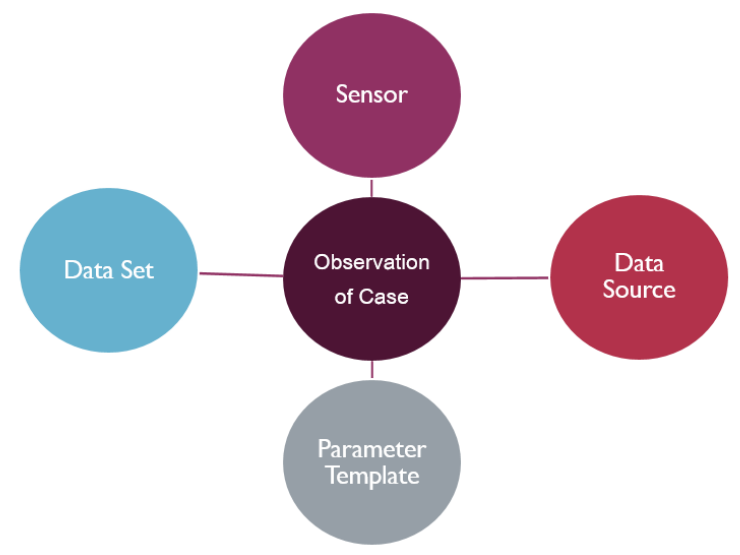

Figure 2. Social sensor design concept

The design of our social sensor has three features: modularity, reusability, and manageability. First, modularity means that each sensor is a reusable module derived from analytical methods. Second, in addition to the reusability resulting from the modular design of social sensors, another aspect of reusability comes from the reuse of case study. Parameter settings in a typical case can be reused through the parameter template mechanism. Third, manageability provides an easy-to-operate analysis environment for 
establishing and managing observed cases, parameter templates, the selection of sensors, and the ability to save and publish the results.

\subsection{System Architecture Design}

The design of our system architecture is shown in Figure 3. We have dismantled a social sensor into components, each of which has its own task and goal. All components collaborate together to construct the whole social sensor architecture. In the following subsections, we will describe each of these components in more detail.

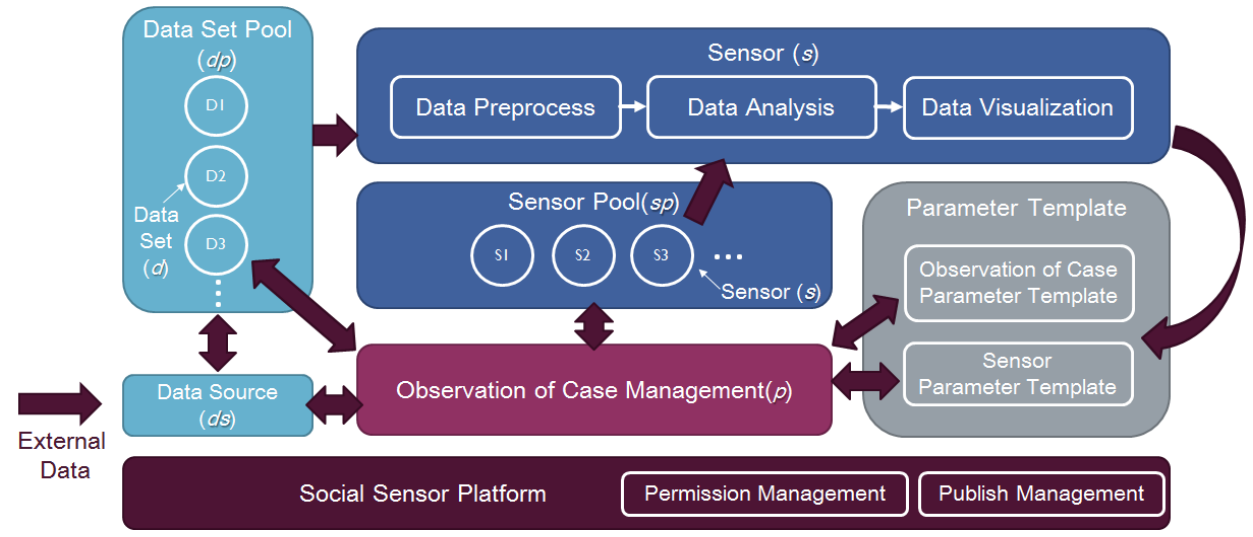

Figure 3. Social sensor architecture design

\subsection{Social Sensor Platform}

Our social sensor platform uses a web-based client-server architecture consisting of three tiers: Presentation, Business Logic, and Data Services. As in most client-server systems, the storage and computing resources are placed at the server side, and the client side usually operates through a browser. Some parts of data visualization computation is handed over to the client side to speed up the operation. In term of software development methodology, we have adopted an object-oriented programming (OOP) paradigm to make the programs reusable, easy to maintain and extendable.

In the platform design, we adopted a role-based authorization mechanism with two types of users: system administrators and researchers. System administrators are responsible for managing system operations while researchers create observation cases, conduct research and publish results through analysis and visualization. In Figure 4, we show snapshots of the implemented platform in action in various stages of the operations of a typical case analysis: the creation of an observation case, case management, sensors on-demand, chart analysis operation, parameter adjustment, sensor setup and tuning, setup of parameter template and other functions. 


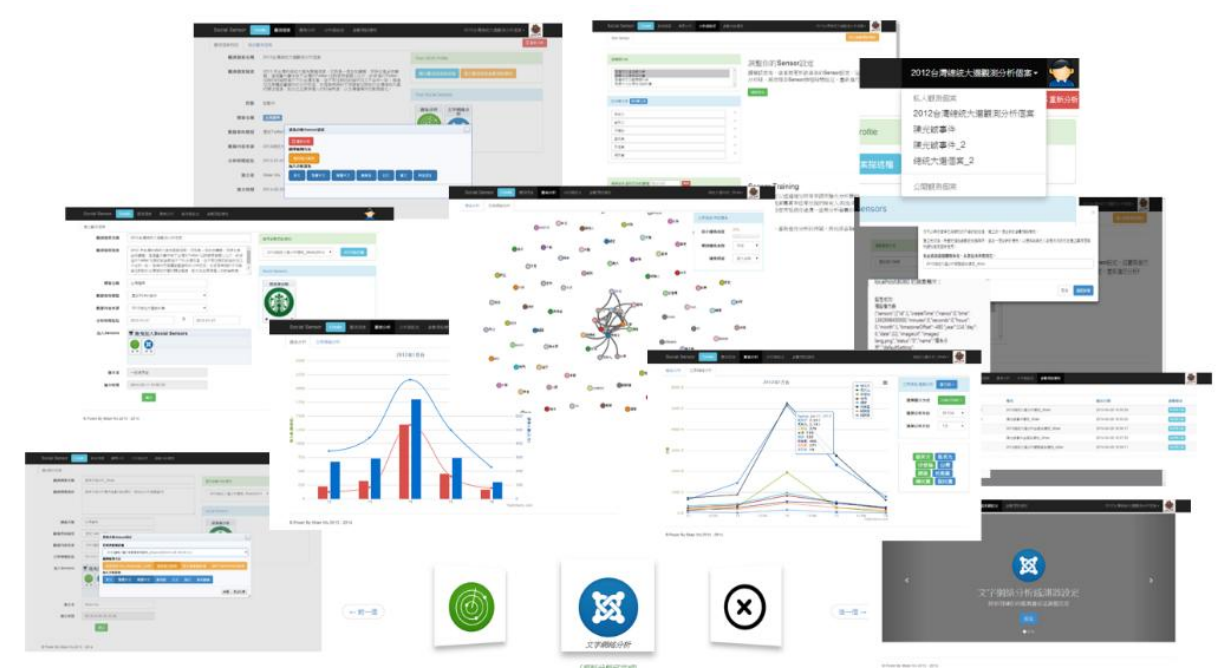

Figure 4. Snapshots of the functions provided in social sensor platform

\subsection{Observation of Case Management and Observation of Cases}

In a typical observation case, researchers usually choose an event as a research theme that they are interested in for further investigation. Assume that a researcher is denoted as $R$, an observation case is denoted as $p$, and $P_{R}$ is defined as the set of cases $p$ 's observed by $R$.

In other words, $P_{R}=\left\{p_{1}, p_{2}, \cdots p_{n-1}, p_{n}\right\}$ represents the cases observed by $R$. An observation case $p$ is defined with several parameters. For example, the data source is denoted by $d s$, the data set is denoted by $d$, the set of social sensors is denoted by $s=\left(s_{1}, s_{2}, \cdots, s_{n-1}, s_{n}\right)$, start and end times of the data set defining the time interval for analysis are denoted by $T_{S}$ and $T_{e}$ respectively. While $d s$ and $d$ are single-value variables, $s$ can have multiple choices. Researchers can ask "what-if" questions by performing experiments of data analysis repeatedly with different sets of parameter settings. When a desired analysis result is acquired, the researchers can save the current settings as a template for use in future cases.

\subsection{Data Sources}

Today, a wide range of social media is available, and their data types and analysis goals are usually different. Data sources refer to the type of social media such as Twitter or Facebook. In this research, we used Twitter as an example of a data source to establish the observation cases. For a given data source, there could be multiple entries of data sets for analysis. 
For example, here are two examples of data sources with multiple data sets associated with: $d s_{1}\left\{d_{1}, d_{2}, d_{3}\right\}$ and $d s_{2}\left\{d_{4}, d_{5}\right\}$.

\subsection{Data Set Pool and Data Sets}

A data set is acquired from a data source through the data collection process with specific query conditions such as hashtags, and time intervals. A data set pool, denoted by $d p$, is a common place for storing data sets in an observation case. In this research, we used a database to store the data sets. In each observation case, one can change the data source by selecting an appropriate social media type and then the data sets from the given data set pool.

\subsection{Sensor Pool and Sensors}

Sensor, denoted by $s$, is an analytical module designed for a specific analytical purpose. Each sensor is designed based on a modular approach so as to facilite use with various analytical methods to satisfy the demands of social media studies. To be self-contained, though not required, a sensor typically is designed with appropriate visualization to facilitate the analysis. Each sensor is independent and does not affect other sensors. Each sensor in the design must include three modules: data preprocessing, data analysis, and data visualization. In addition, the three modules need to be streamlined to avoid any manual external data exchanges.

The design concept of sensors and sensor pool is shown in Figure 5. A sensor $s$ starts the analytical computation by first entering the data preprocessing stage in which one obtains $d$ from $d p$ and then acquires the data in the intervals between $T_{S}$ and $T_{e}$ set in $p$. Then depending on type of analytical method used in the sensor, the desired data features, such as content texts in tweets, will be extracted automatically and sent to the next stage: data analysis. In the data analysis stage, based on the algorithmic design of the sensor, data will be further analyzed and saved back to independent storage for the sensor. In the third stage, the derived data after the analytical processing will be visualized according to the type of data and the design of the sensor. Usually, interactive queries and real-time display are preferred for instant feedback. In addition, since the data analysis stage is the most time consuming stage, the computation in this stage is set in the background by default, such that the users can proceed to visualize previous or partial results while the new analytical results are being processed. 


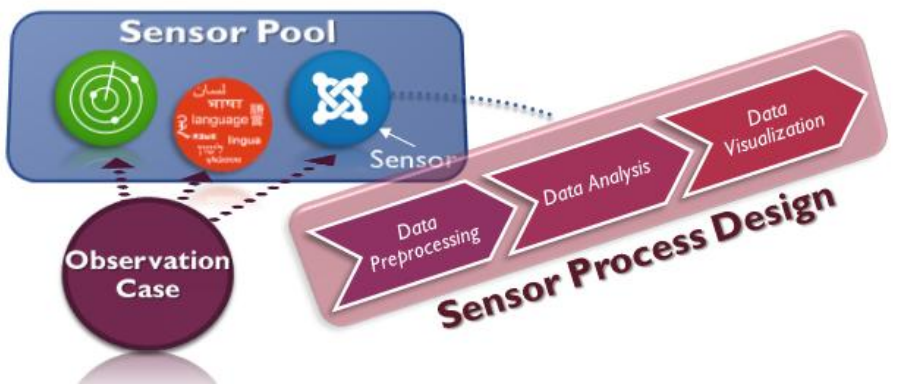

Figure 5. Design concept of sensors and sensor pool

Sensor Pool, denoted by $s p$, is a place to store available sensors $\left\{s_{1}, s_{2}, \cdots, s_{n-1}, s_{n}\right\}$, from which $p$ selects the desired sensors for analysis. Each sensor will be assigned specific parameters that can be adjusted in a given $p$ based on its analytical objective.

\subsection{Parameter Templates}

Once we have the observation cases and sensors parameterizable, for the same case, different researchers may choose different analytical methods, and for the same analytical method, researchers may even choose different parameter settings based on their experience and exploration. In our system, we hope to retain the sensors and parameter settings of a case conducted by a specific researcher in order to replicate the experience of experts and lower barriers to obtaining meaningful analytical results when starting up a new case. In our design, the parameters of a case $p$ can be built into a parameter template, as shown in Figure 6, which includes settings such as $d s$ (data sources), $d$ (data sets), $T_{S}$ (start time for analysis), $T_{e}$ (end time for analysis), the set of selected sensor groups, $\left\{s_{1}, s_{2}, \cdots, s_{n-1}, s_{n}\right\}$, and the parameter settings for each individual sensor. For the last part about the parameters of a sensor, one can choose to save the settings into a parameter template for the sensor for future reuse.

In our system, one can also set the access rights of each individual template (case or sensor) as private or public. The default access setting for a template is private, but can be changed to public access when users consider this appropriate. 


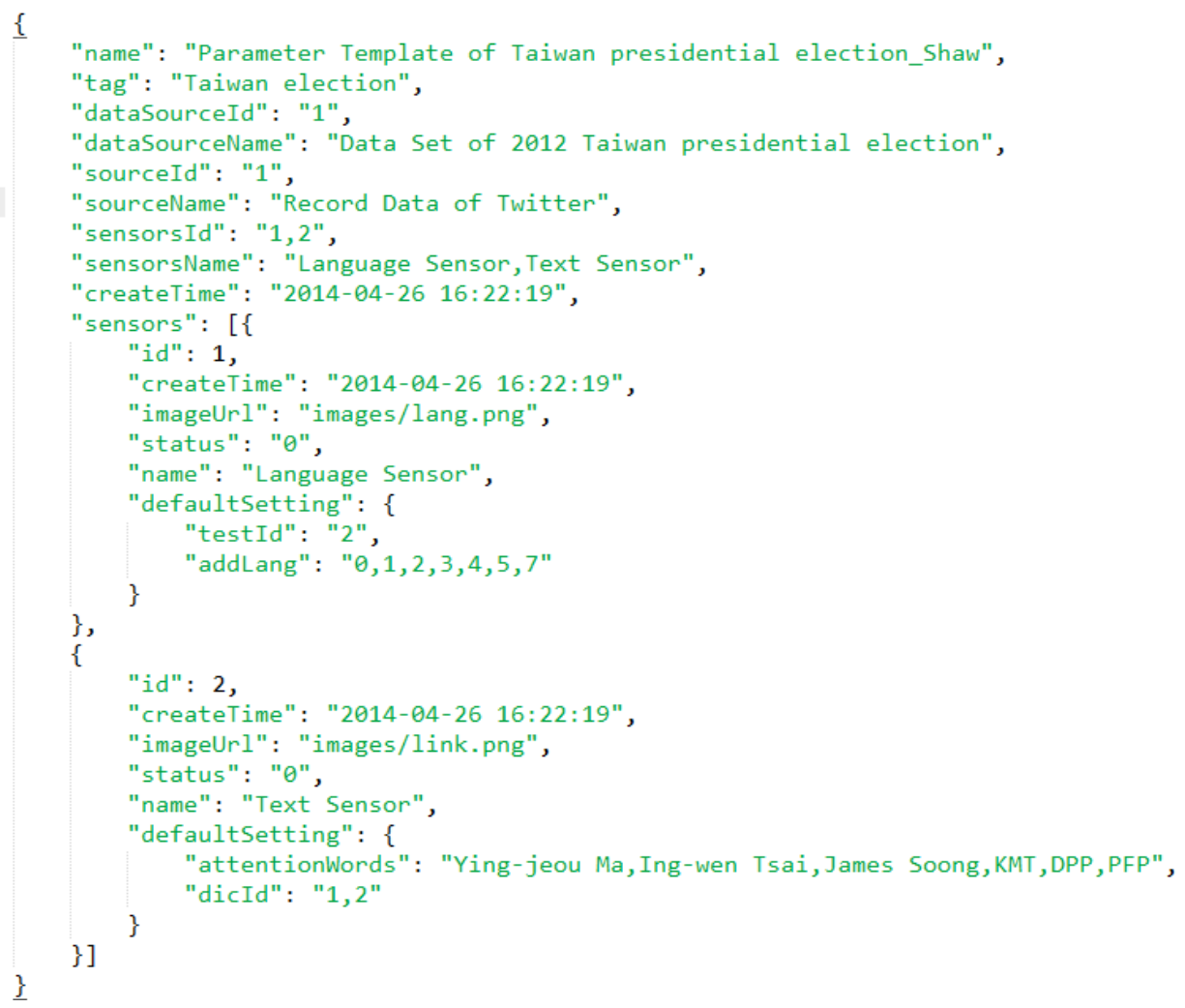

Figure 6. Example of parameter template of an observation case in JSON

\subsection{Language Sensor and Text Sensor}

We implemented two types of sensors: Language Sensor and Text Sensor for demonstration purposes. The language sensor, as shown in Figure 7 , was designed to detect the language used in a tweet. Language sensor can be used for the analysis of tweets about cross-regional discussions, such as about the Taiwan presidential election, umbrella revolution in Hong Kong, anti-China protests in Vietnam, etc. The language sensor can be used to automate the statistical analysis of tweets by different language communities and provides a comparative study. The detection is not trivial because several languages can be used in different parts of a tweet. In the current implementation, the sensor supports language identification for Traditional Chinese (zh-TW), Simplified Chinese (zh), Cantonese (gh), English (en), Japanese (ja), Korean (kr) and other languages. The detection of language used in a tweet can be based on different parts of a tweet such as the entire body content, overall reply post, and @RT/@mention. The system allows a user to customize appropriate parts of the source data in order to conduct language detection. The detection method has been 
implemented and shown to have an average accuracy of 0.94 for inter-coder agreement, and a composite reliability of 0.98 .

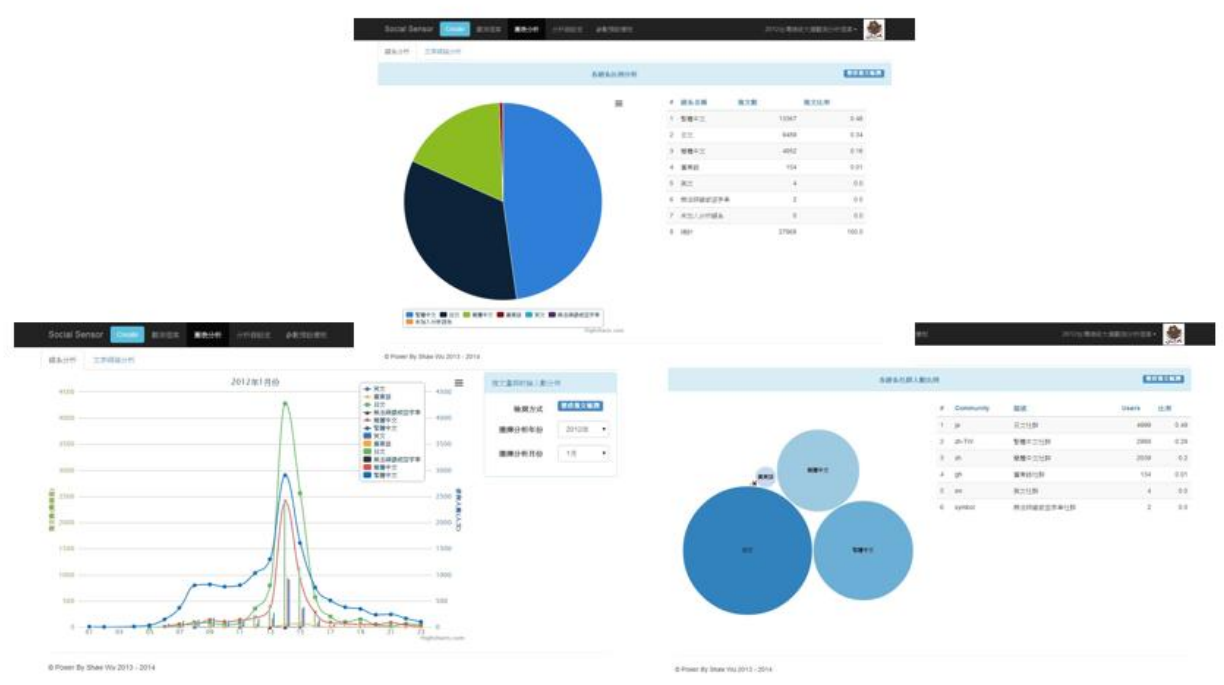

Figure 7. Sample visualization results of the language sensor

Text Sensor, as shown in Figure 8, is another social sensor that has been implemented in the current system. It contains several types of text mining methods, such as segmentation, word frequency statistics, and co-occurrence analysis, for analyzing the text content in a tweet. As an example, these methods can be used to analyze the occurrence of keywords in tweets about changes in an election. The sensor can also be used for time-series analysis on various data items and displays co-occurrence relations in a work network.

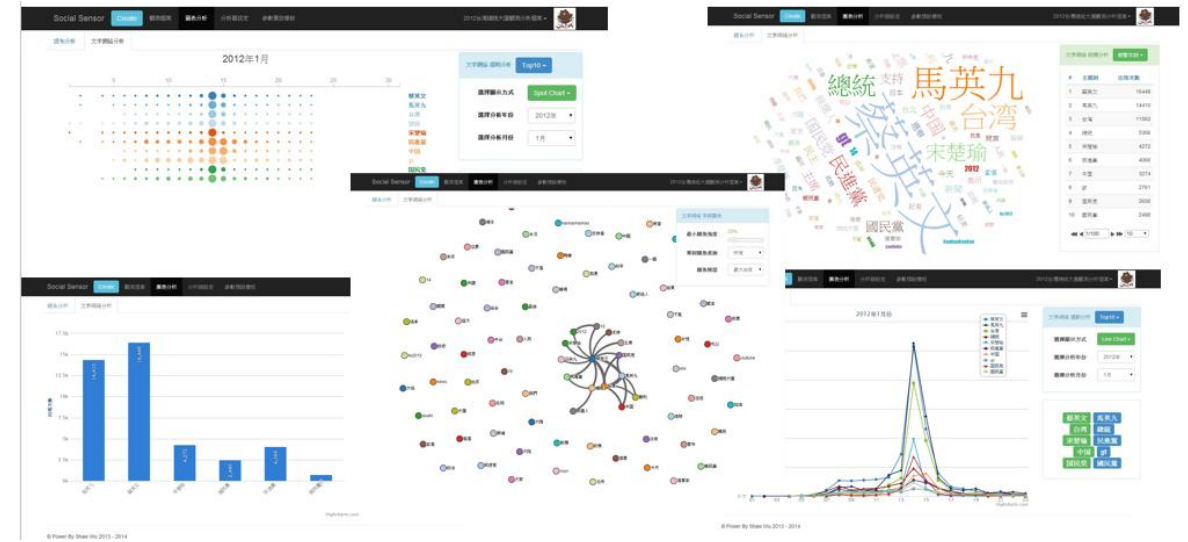

Figure 8. Sample visualization of text sensor results 


\subsection{System Implementation}

The social sensors in this work have been implemented as a web-based system allowing users to do the analysis through a web browser. The underlying language at the server side is Java and a Model-View-Controller (MVC) model based on SSH (Spring, Struts2, Hibernate) was used to design the system as a component-based architecture. At the client side, we have heavily used JavaScript, JQuery and other front-end languages for interactive design, and adopted AJAX technology to communicate between the client side and the server side. In terms of visualization design, we used libraries such as HighCharts, d3.js, and SVG to implement various graphical displays according to the analysis needed in the social sensors.

\section{EXPERIMENTAL EVALUATION}

\subsection{Experimental Design and Evaluation Method}

We designed experiments to evaluate the proposed system. The diary method ${ }^{14}$ was adopted for evaluation and qualitative analysis. In addition to recording the whole operation process by the users, we also designed an unstructured open-ended questionnaire for the participants to reflect upon their user experience. Four users with domain knowledge were invited to participate in the experiment. Users ID A and B were senior analysts, and users $C$ and $D$ were junior analysts. They were asked to use the system three times spread over a period of about one week. The users recorded their steps and processes that they have used to analyze the Taiwan presidential election. At the end of each trial run, the users were asked to fill in a survey with open-ended questions about usability. At the end of the overall experiment, another questionnaire about system usefulness was given to the users. In addition to the qualitative analysis of the recording and feedback by the users, we also conducted a quantitative analysis based on the given textual feedback. The dual method is based on the concept of a ladder of analytical abstraction from ${ }^{15,16}$.

\subsection{Evaluation of Usefulness}

On the evaluation of the usefulness of the system, positive, fair, and negative comments are $60.32 \%, 31.75 \%$, and $7.94 \%$, respectively, showing that the participants consider most of the functions of the system useful. The descriptive codes of the positive comments are mostly "sensors", "text sensor", "parameter template", "useful", "time-saving", "quick analysis", etc. We have designed a coding scheme to further analyze the collected comments. The codes are classified into three categories: keynote, function, and feeling codes. By the coding path method as shown in Figure 9, each comment was coded with at least two codes out of three. For example, 
the coding path of "A0A" (sensors $\rightarrow$ null $\rightarrow$ useful) means that the sensors were considered useful. With this method, we can observe the large amount of feedback text in a quantitative way. In Table 1, we can also find evidence for positive feedback about the system design.

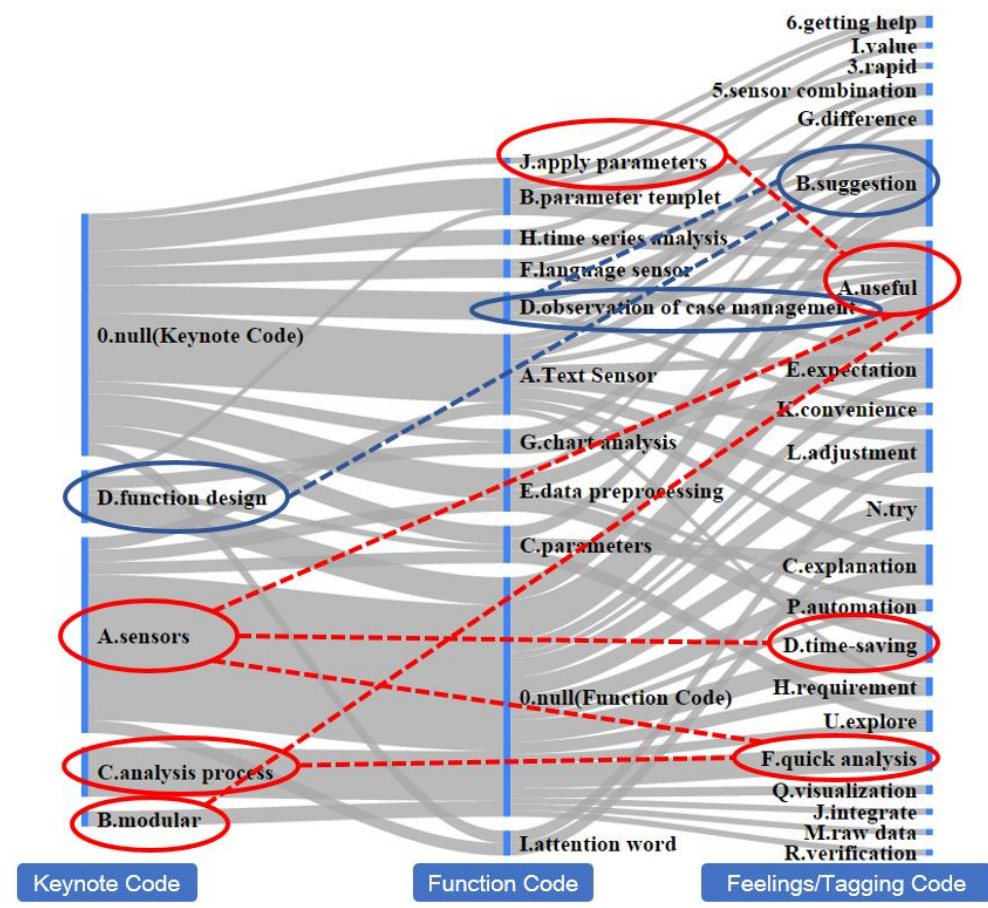

Figure 9. Coding system for system usefulness

\subsection{Evaluation of Modularity}

Table 1 indicates that the modularity of the system was well received and appreciated by the users. The frequency of coding path B0A, together with others, indicates that the sensor modularity was useful, time-saving and provides rapid analysis. Subject A mentioned that the language analysis sensor was important for analyzing tweets in mainland China, Taiwan and Hong Kong, but not necessarily applicable to other cases. Providing a pluggable social sensor allows the users to select appropriate modules in different analysis cases. Subject B considered the modular design of the system as helpful for users to effectively learn the tools available and to control tool variables. Subject D considered the system as a time-saving tool for self-exploration and data processing. Subjects A and C mentioned their desire to see more sensors developed. In short, modular design of the system was considered useful, time-saving, and flexible for future integration of new sensors. 
Table 1. Top-ten for coding path

\begin{tabular}{|c|c|c|c|c|c|c|c|c|}
\hline $\begin{array}{l}\text { Coding } \\
\text { Path }\end{array}$ & $\begin{array}{l}\text { Keynote } \\
\text { Code Id }\end{array}$ & Keynote Code & $\begin{array}{c}\text { Function } \\
\text { Code Id }\end{array}$ & Function Code & $\begin{array}{c}\text { Feelings/ } \\
\text { Comment } \\
\text { Code Id }\end{array}$ & $\begin{array}{c}\text { Feelings/ } \\
\text { Tagging Code }\end{array}$ & $\begin{array}{l}\text { Path } \\
\text { Count }\end{array}$ & $\begin{array}{l}\text { Occurrence } \\
\text { Frequency }\end{array}$ \\
\hline $\mathrm{A} 0 \mathrm{~A}$ & A & sensors & 0 & null & $\mathrm{A}$ & useful & 8 & $4.47 \%$ \\
\hline B0A & B & modular & 0 & null & A & useful & 5 & $2.79 \%$ \\
\hline A0D & A & sensors & 0 & null & $\mathrm{D}$ & time-saving & 5 & $2.79 \%$ \\
\hline D0B & $\mathrm{D}$ & function design & 0 & null & B & suggestion & 5 & $2.79 \%$ \\
\hline $\mathrm{A} 0 \mathrm{~F}$ & A & sensors & 0 & null & $\mathrm{F}$ & quick analysis & 5 & $2.79 \%$ \\
\hline ODB & 0 & null & $\mathrm{D}$ & observation of case management & B & suggestion & 4 & $2.23 \%$ \\
\hline OBA & 0 & null & $\mathrm{B}$ & parameter template & A & useful & 4 & $2.23 \%$ \\
\hline $\mathrm{A} 0 \mathrm{~N}$ & A & sensors & 0 & null & $\mathrm{N}$ & try & 4 & $2.23 \%$ \\
\hline $\mathrm{A} 0 \mathrm{~L}$ & A & sensors & 0 & null & $\mathrm{L}$ & adjustment & 4 & $2.23 \%$ \\
\hline $\mathrm{COF}$ & $\mathrm{C}$ & analysis process & 0 & null & $\mathrm{F}$ & quick analysis & 3 & $1.68 \%$ \\
\hline
\end{tabular}

\subsection{Evaluation of Reusability}

The system attempts to use parameter templates to facilitate replication of the analysis experience from previous case studies. Basically, all four users consider the reusability through parameter template as a useful feature. The coding paths D0B and OBA reveal that using the parameter template was indeed useful. Subject A said that, "Using the default parameter values as a starter allows users to reduce initial trial-and-error." Subject D mentioned that "The basis of corpus are rather common, and therefore it is a good idea to use the default parameters in a sensor to prevent the users from forgetting to adjust the parameters." There were also some suggestions on the feature. Subject $\mathrm{C}$ suggested that the format of the parameter settings can be improved for better readability. A better authoring interface was also more desirable.

\subsection{Evaluation of Manageability}

For manageability, all subjects agreed that the system indeed can provide effective management of case studies. The coding paths of A0A, $\mathrm{A} 0 \mathrm{D}, 0 \mathrm{DB}, \mathrm{A} 0 \mathrm{~N}, \mathrm{~A} 0 \mathrm{~L}$, and $\mathrm{COF}$ were all examples of comments with positive feedback on case management. Nevertheless, there were also suggestions for improvement. For example, subject A suggested that the system should provide a data export function to allow further analysis in other advanced software packages. Subject $\mathrm{C}$ thought that saving analytical parameters for a case was more desirable than saving the analysis results. Subject C suggested implementation of a function for personal analysis history. In short, the manageability feature of the system can significantly reduce the time needed to prepare and manage a data analysis case. Suggestions were also given to increase interconnection interfaces with other analysis tools and to improve the management functions for case studies. 


\section{CONCLUSIONS AND FUTURE WORK}

In this research, we proposed the concept of a social sensor for data analytics of social media and implemented a system to realize this concept with the design goals of manageability, modularity, and reusability. In this system, a user can quickly perform data analysis and manage a case study by specifying data sources, adjusting case parameters, and visualize the result immediately. One can perform a case study by choosing appropriate social sensors that are extensible as a plug-in. The experience of appropriate parameter settings for a case can also be saved into a parameter template for reuse in other similar case studies. In addition, this system features a modularized design allowing for different analytical modules that are reusable in different sensors and cases. As examples, a language sensor and a text sensor were implemented. In the language sensor, we proposed a method to distinguish the main language used in a tweet. The method had an average accuracy of 0.94 for Intercoder Agreement (IA), and 0.98 for Composite Reliability (CR).

System evaluation was done with qualitative analysis through the diary method. The evaluation results for usefulness, modularity, reusability, and manageability were all very positive. The results also show that this tool can greatly reduce the time needed to perform data analysis and solve problems encountered in traditional analysis process. The experimental results support the concept of a social sensor and the proposed system design was useful in the data analysis process for social media.

In the future, we hope to improve the interface design and operational flow of the system. We would also like to improve the design of existing social sensors and increase the interactivity among the sensors. We also hope to handle more types of social media and accumulate more case experiences. Future studies can investigate more case studies of major events such as the umbrella revolution in Hong Kong, anti-China protests in Vietnam, and related events in China, Japan, South Korea, etc.

\section{REFERENCES}

[1] B. Lorica, Big Data Now: 2013 Edition. USA: O'Reilly Media Publishing, 2013.

[2] Y.-C. Cheng, and P.-L. Chen, Emerging communities in social media during the 2012 Taiwanese presidential elections: A big-data analysis approach. Mass Communication Research, 120, 121-165, 2014.

[3] A.M. Kaplan, and M. Haenlein, Users of the world, unite! The challenges and opportunities of social media. Business horizons, 53(1), 59-68, 2010. http://dx.doi.org/10.1016/j.bushor.2009.09.003.

[4] OECD., Participative web and user-created content: Web 2.0, wikis, 
and social networking. Paris: Organisation for Economic Co-operation and Development, 2007.

[5] Lon S. Cohen, Is there a difference between social media and social networking? Retrieved on April 30, 2009, from http://lonscohen.com/blog/2009/04/difference-between-social-media-a nd-social-networking.

[6] E.J. Yuan, M. Feng, and J.A. Danowski, "Privacy" in semantic networks on Chinese social media: The case of Sina Weibo. Journal of Communication, 63(6), 1011-1031, 2013. http://dx.doi.org/10.1111/jcom.12058.

[7] H. Kwak, C. Lee, and S. Moon. What is Twitter, a social network or a news media? Paper presented at the 19th International Conference on World Wide Web, Raleigh, NC, USA, April 26-30, 2010. http://dx.doi.org/10.1145/1772690.1772751.

[8] J. Burgess, and A. Bruns, (Not) the Twitter election. Journalism $\begin{array}{lll}\text { Practice, } & \text { 6(3), 384-402, }\end{array}$ http://dx.doi.org/10.1080/17512786.2012.663610.

[9] S. Vieweg, A.L. Hughes, K. Starbird, and L. Palen, Microblogging during two natural hazards events: What twitter may contribute to situational awareness. Paper presented at the SIGCHI Conference on Human Factors in Computing Systems, Atlanta, GA, USA, April 10-15, 2010. http://dx.doi.org/10.1145/1753326.1753486.

[10] S.-F. Shih, Information transmission analysis and automated classification design for new media in a disaster event - case study of typhoon Morakot. Master Thesis, Department of Computer Science, National Chengchi University, Taiwan, 2013.

[11] V. Vasudevan, J. Wickramasuriya, S. Zhao, and L. Zhong, Is Twitter a good enough social sensor for sports TV? Paper presented at the 2013 IEEE International Conference on Pervasive Computing and Communications Workshops, San Diego, CA, USA, March 18-22, 2013. http://dx.doi.org/10.1109/PerComW.2013.6529478.

[12] T. Sakaki, M. Okazaki, Y. Matsuo, Earthquake shakes Twitter users: Real-time event detection by social sensors. Paper presented at the 19th International Conference on World Wide Web, Raleigh, NC, USA, April 26-30, 2010. http://dx.doi.org/10.1145/1772690.1772777.

[13] T. Sakaki, Y. Matsuo, T. Yanagihara, N.P. Chandrasiri, and K. Nawa, Real-time event extraction for driving information. Paper presented at the 2012 IEEE International Conference on Cyber Technology in Automation, Control and Intelligent Systems, Bangkok, Thailand, May 27-31, 2012. http://dx.doi.org/10.1109/CYBER.2012.6392557.

[14] S.-M. Wang, ICTs and everyday rhythms: Looking into the Methods. 
Journal of Communication Research and Practice, 4(1), 23-43, 2014.

[15] T.F. Carney, Collaborative inquiry methodology. Windsor, Ontario, Canada: University of Windsor, Division for Instructional Development, 1990.

[16] F.F. Chang, The five steps of qualitative data analysis: Climbing up a ladder of abstraction. Journal of Elementary Education, 35, 87-120, 2010. 